\title{
Domain shuffling and the evolution of vertebrates
}

\author{
Takeshi Kawashima, ${ }^{1,2,3,9}$ Shuichi Kawashima, ${ }^{4}$ Chisaki Tanaka, ${ }^{5}$ Miho Murai, ${ }^{6}$ \\ Masahiko Yoneda, ${ }^{6}$ Nicholas H. Putnam, ${ }^{2,7}$ Daniel S. Rokhsar, ${ }^{2,7}$ Minoru Kanehisa, ${ }^{4,8}$ \\ Nori Satoh, ${ }^{1}$ and Hiroshi Wada ${ }^{5,9}$
}

${ }^{1}$ Okinawa Institute of Science and Technology, Uruma, Okinawa 904-2234, Japan; ${ }^{2}$ Department of Energy Joint Genome Institute, Walnut Creek, California 94598, USA; ${ }^{3}$ Japanese Society for Promotion of Sciences, Tokyo 102-8471, Japan; ${ }^{4}$ Human Genome Center, Institute of Medical Science, University of Tokyo, Tokyo 108-8639, Japan; ${ }^{5}$ Graduate School of Life and Environmental Sciences, University of Tsukuba, Tsukuba 305-8572, Japan; ${ }^{6}$ Department of Nursing \& Health, School of Nursing \& Health, Aichi Prefectural University, Nagoya 463-8502, Japan; ${ }^{7}$ Center for Integrative Genomics, University of California, Berkeley, Berkeley, California 94720 , USA; ${ }^{8}$ Bioinformatics Center, Institute for Chemical Research, Kyoto University, Gokasho, Uji, Kyoto 611-0011, Japan

\begin{abstract}
The evolution of vertebrates has included a number of important events: the development of cartilage, the immune system, and complicated craniofacial structures. Here, we examine domain shuffling as one of the mechanisms that contributes novel genetic material required for vertebrate evolution. We mapped domain-shuffling events during the evolution of deuterostomes with a focus on how domain shuffling contributed to the evolution of vertebrate- and chordate-specific characteristics. We identified $\sim 1000$ new domain pairs in the vertebrate lineage, including $\sim 100$ that were shared by all seven of the vertebrate species examined. Some of these pairs occur in the protein components of vertebrate-specific structures, such as cartilage and the inner ear, suggesting that domain shuffling made a marked contribution to the evolution of vertebrate-specific characteristics. The evolutionary history of the domain pairs is traceable; for example, the Xlink domain of aggrecan, one of the major components of cartilage, was originally utilized as a functional domain of a surface molecule of blood cells in protochordate ancestors, and it was recruited by the protein of the matrix component of cartilage in the vertebrate ancestor. We also identified genes that were created as a result of domain shuffling in ancestral chordates. Some of these are involved in the functions of chordate structures, such as the endostyle, Reissner's fiber of the neural tube, and the notochord. Our analyses shed new light on the role of domain shuffling, especially in the evolution of vertebrates and chordates.
\end{abstract}

[Supplemental material is available online at www.genome.org.]

The question of how novel structures are created by changing genetic information is one of the most challenging issues in evolutionary biology. It is generally accepted that the morphological features of various multicellular animals are built on a common set of genes. Carroll et al. (2001) and Davidson (2006) proposed that the novel features emerged as a result of altered gene expression patterns. Novel genetic material has also contributed to the evolution of novel structures. Much attention has been focused on gene duplications as a mechanism for the evolution of novel genetic material. Particularly in the case of vertebrate evolution, whole genome duplications that occurred twice in ancestral vertebrates have been regarded as the main force driving the evolution of novel structures (Holland et al. 1994). As an additional mechanism, we focus here on domain shuffling (Chothia et al. 2003; Babushok et al. 2007). Several different molecular mechanisms for domain shuffling have been proposed. Since the domains are often correlated with exon boundaries, exon shuffling is believed to be one of the major forces driving domain shuffling (Liu and Grigoriev 2004). In addition, the introns at the boundaries of domains show a marked excess of symmetrical phase combinations, and, consequently, these domains may be inserted without changing the reading frame of ancestral genes (Kaessmann et al. 2002; Vibranovski et al. 2005). Some other

\footnotetext{
${ }^{9}$ Corresponding authors.
}

E-mail 98champ@msg.biglobe.ne.jp; fax 81-29-853-4671.

E-mail takeshik@oist.jp; fax 81-98-934-5622.

Article published online before print. Article and publication date are at http://www.genome.org/cgi/doi/10.1101/gr.087072.108. mechanisms might have been involved in domain shuffling, such as the simple fusion of genes and recruitment of mobile elements (Babushok et al. 2007; Ekman et al. 2007).

In contrast to thorough investigation of the mechanistic aspects of domain shuffling, the contribution of the new genes created by domain shuffling to the evolution of phenotype has not been sufficiently explored. Because domain shuffling occurs more frequently in metazoan lineages than in unicellular organisms, suggesting that domain shuffling played an important role in the evolution of multicellularity (Patthy 2003; Ekman et al. 2007), we reasoned that domain shuffling also contributed to the elaboration of metazoan body plans. In this study, we comprehensively investigated the domain shuffling events during deuterostome evolution. The complete amphioxus genome sequence provides sufficient deuterostome genomic sequences (Dehal et al. 2002; Sea Urchin Genome Sequencing Consortium 2006; Putnam et al. 2008) to map the domain shuffling events accurately. We listed gene models that were created by domain shuffling in the ancestors of vertebrates, and examined how domain shuffling contributed to the evolution of new genes for vertebrate-specific characteristics. The contribution of domain shuffling was also examined in the evolution of chordates.

\section{Results and Discussion}

Detection of domain shuffling in deuterostome evolution

We identified pairs of protein domains found in single-gene models, including the human, mouse, rat, chicken, frog, pufferfish, 
zebrafish, ascidian, and amphioxus genomes. We took the order of the domains into account, so that the domain pair A and B (in order from the $\mathrm{N}$ terminus) was treated as a distinct pair from domains in the order B and A. This is because these domain pairs with different orders are more likely to have been gained by distinct domain shuffling events than by divergence from a common ancestral pair. We surveyed domain pairs found in all splicing variants in order to detect all domain pairs transcribed in the genomes. As outgroups, domain pairs from the proteomes of a sea urchin, fly, mosquito, gastropod snail, two species of nematode, sea anemone, choanoflagellate, slime mold, yeast, and plant (Arabidopsis) were obtained (Supplemental Table 1). In the 20 proteomes, profile-HMM (HMMER) searches of the Pfam database ( $E$-value cutoff of $10^{-3}$; see Methods) recognized 4649 protein domains. Of these, 2727 were found paired with other domains. Approximately 2000-5500 domain pairs were found in the gene models of each species. For example, 4057 domain pairs were found in the human gene models, with 2251 found in ascidian and 5387 in amphioxus. From these datasets, we surveyed domain pairs shared by the major deuterostome taxa (Table 1). By this method, triplets of domains were detected as two or three domain pairs in one gene model (e.g., SNF2 histone linker PHD RING helicase or attractin in Table 2).

Table 1. Shared domain combinations and unique domains

\begin{tabular}{|c|c|c|c|}
\hline & $\begin{array}{c}\text { Class I } \\
\text { combinations }{ }^{a}\end{array}$ & $\begin{array}{c}\text { Class II } \\
\text { combinations }\end{array}$ & $\begin{array}{c}\text { Unique } \\
\text { domains }\end{array}$ \\
\hline VCBSO & 0 & 1326 & 1532 \\
\hline VCBS & 0 & 20 & 2 \\
\hline VCB_O & 0 & 355 & 116 \\
\hline VC_SOO & 0 & 129 & 25 \\
\hline V_BSO & 0 & 458 & 161 \\
\hline CBSO & 0 & 15 & 0 \\
\hline$\overline{\mathrm{V}} \mathrm{CB}$ & 0 & 43 & 5 \\
\hline VC_ $\overline{S_{-}}$ & 2 & 4 & 1 \\
\hline V_BS & 2 & 51 & 4 \\
\hline $\mathrm{CBS}_{-}^{-}$ & 0 & 8 & 0 \\
\hline $\bar{V} C \_-\bar{O}$ & 0 & 143 & 11 \\
\hline V_B_O & 0 & 496 & 83 \\
\hline CB_O & 0 & 28 & 2 \\
\hline$\overline{\mathrm{V}} \mathrm{SO}$ & 0 & 131 & 25 \\
\hline C_C_SO & 0 & 4 & 0 \\
\hline $\bar{B} \mathrm{BSO}$ & 0 & 92 & 10 \\
\hline$\overline{\mathrm{VC}}$ & 1 & 34 & 3 \\
\hline V_B & 21 & 157 & 18 \\
\hline${ }_{C} B_{-}$ & 0 & 35 & 0 \\
\hline $\bar{v}_{-} \bar{S}_{-}$ & 2 & 43 & 1 \\
\hline $\bar{C}_{-} S_{-}$ & 0 & 4 & 0 \\
\hline $\mathrm{BS}$ & 2 & 71 & 1 \\
\hline$\overline{\mathrm{V}} \quad \overline{\mathrm{O}}$ & 0 & 439 & 118 \\
\hline $\bar{C} \mathrm{O}$ & 0 & 21 & 2 \\
\hline B_O & 0 & 242 & 25 \\
\hline SO & 0 & 44 & 3 \\
\hline $\bar{V}$ & 368 & 859 & 209 \\
\hline $\bar{C}$ & 0 & 79 & 0 \\
\hline$B$ & 32 & 1933 & 7 \\
\hline$\overline{S_{-}}$ & 10 & 455 & 2 \\
\hline 0 & 0 & 2951 & 361 \\
\hline
\end{tabular}

${ }^{a}$ Number of shared domain combinations for which one of the domains was found only in that species.

${ }^{b}$ Number of shared domain combinations for which the domains were found in other species.

${ }^{c}$ Numbers of domains uniquely found in the species.

(V) Vertebrates; (C) Ciona (ascidian); (B) Branchiostoma (amphioxus); (S) Strongylocentrotus (sea urchin); (O) other species examined.
Based on the table of shared domain pairs (Table 1), we mapped the gained and lost domain pairs on a phylogenetic tree that had been previously deduced by using concatenated amino acid sequences (Fig. 1A; Putnam et al. 2008). If more than two taxa shared domain pairs, we regarded the pairs as having been acquired in the last common ancestor. If pairs were shared in only some of the descendent taxa, we assumed that those pairs were lost in the rest of the descendent taxa. This method to reconstruct the gain/loss of domain pairs does not follow the maximum parsimony principle. Rather, to filter domain pairs that are more likely to have been achieved by independent domain shuffling events, we examined the overall domain structures of the gene models and defined a convergent index (CI) (see Methods). Although these independent acquisitions were thought to be rare (Gough 2005), we detected several domain pairs that are likely to have been achieved independently. By filtering domain pairs of $\mathrm{CI} \geq 0.5$, we found that 15 pairs that were shared by the sea urchin and some chordates had rather different domain architectures (Supplemental Fig. 1). Therefore, these domain pairs were more likely to have been acquired by convergence. Among 269 domain pairs that were assumed to have been gained in the ancestors of chordates, 13 pairs showed a $\mathrm{CI} \geq 0.5$, and thus were suggested to have evolved convergently. No domain pairs shared by ascidians and vertebrates, and new pairs acquired in the vertebrate lineage, showed a $\mathrm{CI} \geq 0.5$.

After filtering domain pairs of $\mathrm{CI}<0.5,209$ pairs were assumed to have been acquired in the common ancestors of deuterostomes, 256 pairs in the common ancestors of chordates, and 35 in the common ancestors of ascidian and vertebrates. Regarding the gained and lost pairs in each deuterostome taxon, it is notable that many of the new pairs (1965 pairs) were acquired in the amphioxus lineage, which exceeded the number observed in the vertebrate lineage. Because some ambiguity remains in the gene models predicted from the genome sequences and EST sequences, some of the predicted gene models may combine two distinct transcripts. If these misassigned gene models are abundant in the amphioxus genome, the number of amphioxusspecific domain pairs may be artifactually high. However, we think it is not the case here, because after filtering of the gene models by EST evidence (gene models supported by stretches of EST sequences and thus proven presence of the transcripts), 847 amphioxus-specific domain pairs were still found, while only 35,280 Pfam domains were recognized in the filtered gene models compared with 121,370 domains recognized in all amphioxus gene models (Supplemental Table 2). Therefore, we think this observation is unlikely to be an artifact of gene modeling. In amphioxus-specific domain pairs, Lectin_C, LRR_1, TPR_1, and TPR_2 occurred most frequently together with other domains, which also frequently were involved in domain shuffling in other lineages. On the other hand, some domains such as Death and F5_F8_type_C were more frequently involved in amphioxus compared with other lineages. Death is involved in apoptosis, and F5_F8_type_C is found in membrane protein or coagulation factors. This is consistent with previous reports that the gene repertoires for apoptosis and the innate immune system are expanded in amphioxus (Holland et al. 2008; Huang et al. 2008). Conversely, many of the pairs were lost in the ascidian lineage, which is not surprising considering that extensive gene loss has occurred in that lineage (Dehal et al. 2002). To correlate genomic complexity with phenotypic variation, it is worth noting that amphioxus species, which have more or less retained their ancestral morphology, acquired several new domain pairs, whereas

\section{Genome Research www.genome.org}




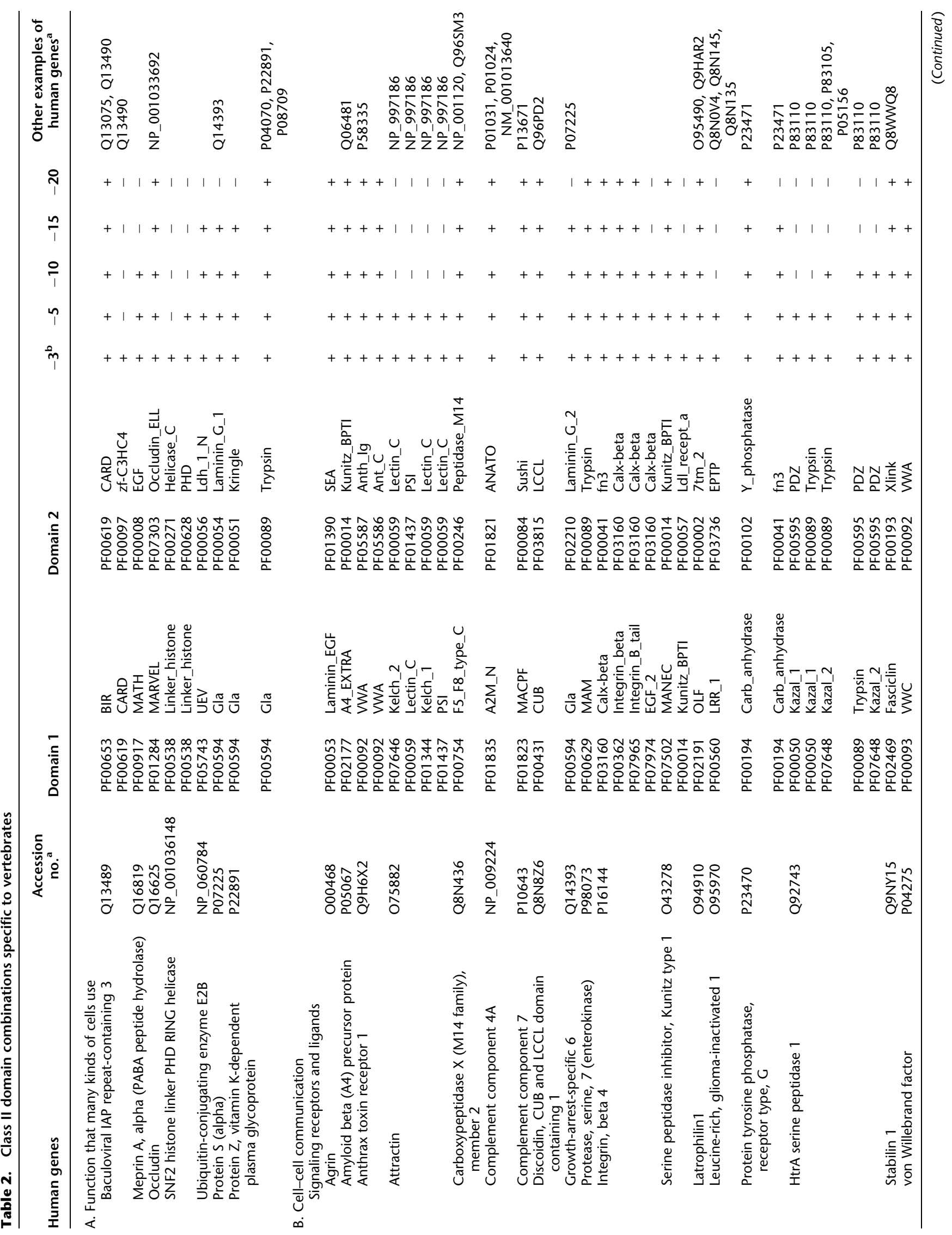




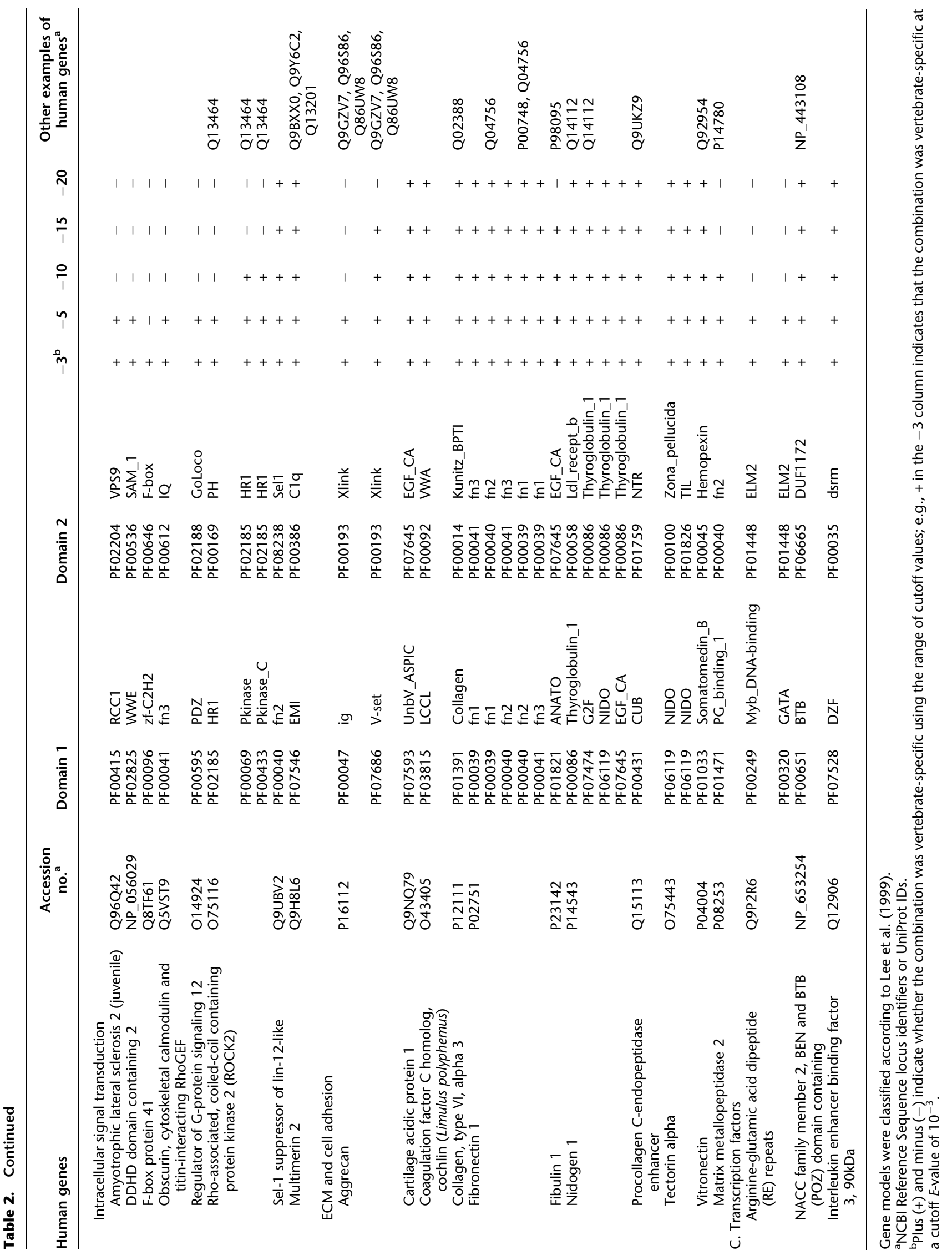




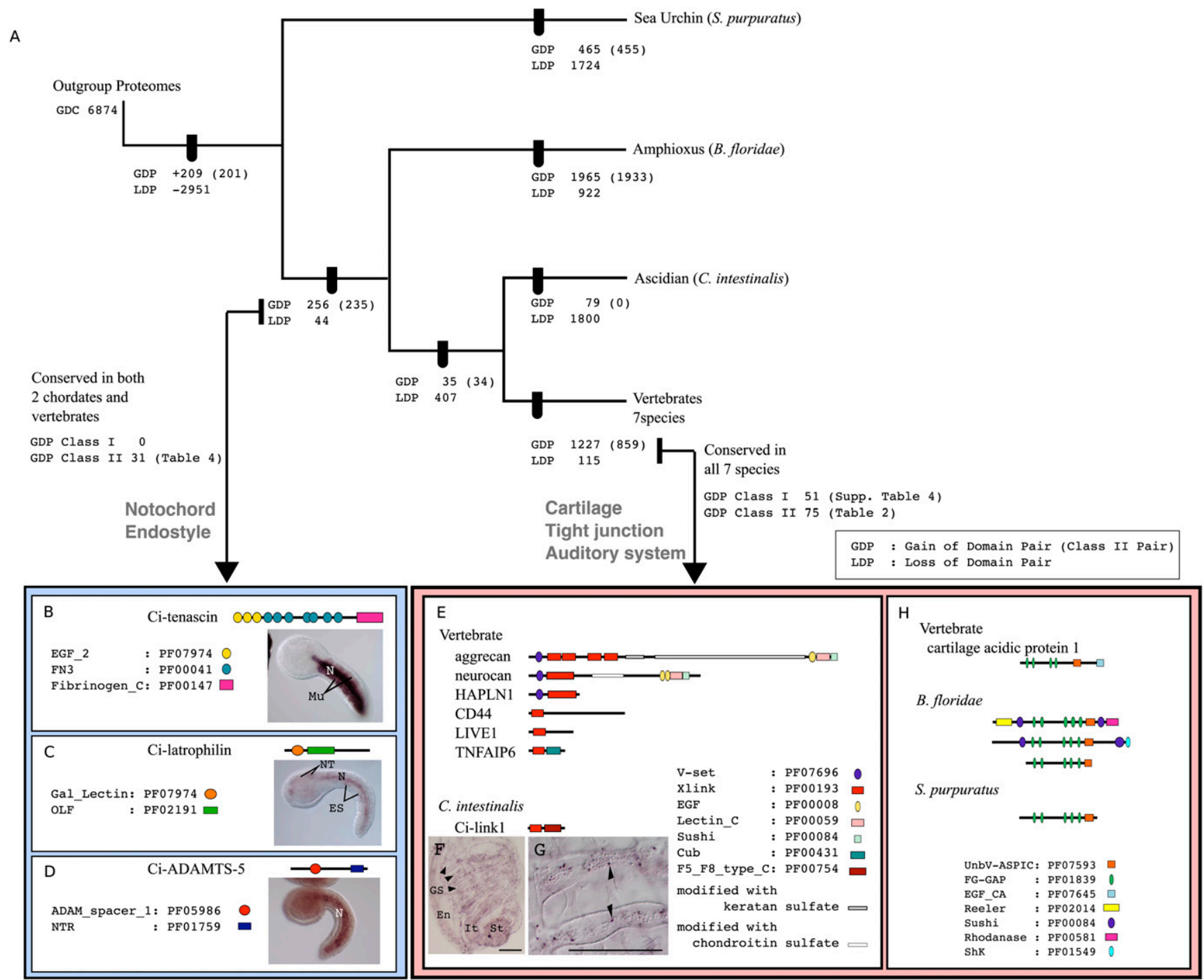

Figure 1. Domain shuffling during the evolution of deuterostomes. (A) Gain and loss of the domain pairs were mapped on the deuterostome phylogenetic tree constructed using molecular phylogenetic analyses of multiple gene sequences (Putnam et al. 2008). Numbers on each node present gains and loss of domain pairs after screening $\mathrm{Cl}<0.5$ (see Methods for the definition of $\mathrm{Cl}$ ). Gains of Class II domain pairs are shown in brackets. (Arrows) Domain pairs acquired in the ancestral chordates, and those in ancestral vertebrates, that were examined in detail. Among 1227 pairs that were acquired in the ancestral vertebrates, 51 class I domain pairs and 75 class II domain pairs were conserved in seven vertebrate species examined. Some of these were involved in vertebrate-specific characters such as cartilage $(E-H)$, tight junctions, and auditory systems (see text for details). Among 256 domain pairs unique to the chordates, 31 pairs were conserved in ascidian, amphioxus, and more than one species of vertebrates. Some of them were involved in chordate-specific characters such as the notochord $(B-D)$ and endostyle (see text for details). $(B-D)$ Tenascin, latrophilin, and $A D A M T S-5$, which were created by domain shuffling in ancestral chordates, are expressed in the notochords of ascidian embryos. Symbols are indicated with the domain ID and Pfam accession numbers (Finn et al. 2006). (B) Ci-tenascin is expressed in the ascidian notochord ( $\mathrm{N}$ ) and muscle cells (Mu). (C) Ci-latrophilin expression was detected in the notochord, neural tube (NT), and endodermal strand (ES) of ascidian larvae. (D) Expression of Ci-ADAMTS-5 was restricted to the ascidian notochord. (E) Domain structures of the proteins that include an Xlink domain. $(F, G)$ Expression of $C i$-link 1 was observed in some of the blood cells from an ascidian juvenile. (Arrowheads) Some of the Ci-link1-positive cells. Scale bars, $50 \mu \mathrm{m}$. (En) Endostyle, (GS) gill slits, (St) stomach, (It) intestine. $(H)$ Domain structures of cartilage acidic protein and proteins containing an ASPIC-and-UnbV domain encoded by amphioxus gene models.

many pairs were lost in the ascidian lineage, which has evolved extensive morphological variation.

\section{Domain shuffling in the ancestral vertebrates}

The vertebrate lineage acquired 1227 domain pairs. Of these, 137 pairs were conserved among all seven species, whereas the rest were restricted to certain taxa (Fig. 1A). Surveying the NCBI Conserved Domain Database (CDD) with each domain pair using
CDART searches (http://www.ncbi.nlm.nih.gov/Structure/cdd/cdd. shtml) identified 11 of the pairs in invertebrates such as bees, Drosophila, or Caenorhabditis elegans (Supplemental Table 3; some genes from Drosophila or other species in the CDD were missing from the proteome database because the gene models were incomplete). Of the remaining 126 pairs, 51 contained one or two domains found only in vertebrate genomes (Supplemental Table 4). These pairs, which we designated as class I domain pairs, therefore may not have evolved via domain shuffling, but instead 
may have resulted from de novo establishment of new domain sequences. Alternatively, some domain sequences might be too divergent to be detected in outgroups, and may not be novel combinations. In contrast, the remaining 75 pairs consisted of two domains, both of which were found in invertebrates as well as in vertebrates (Table 2). We designate them as class II domain pairs, which certainly evolved via domain shuffling. Only two gene models contain both class I and class II domain pairs (latrophilin1 and amyloid beta [A4] protein precursor)

We first asked whether these class II domain pairs were acquired by exon shuffling events. We examined the exon-intron structures of 75 pairs that were found in 143 human gene models. In $24 \%$ of these gene models, the two domains are encoded by exons separated by one intron (referred to as "neighbor" in Fig. 2 and Supplemental Table 5), including those in which either domain is encoded by more than two exons. In $65 \%$, the two domains were encoded by exons separated by two or more introns and one or more exons ("farther" in Fig. 2 and Supplemental Table 5). In only $11 \%$, the two domains were not separated by any introns and, thus, there is an exon that codes at least part of both domains ("same" in Fig. 2, Supplemental Table 5). Although in some domains the boundary of the exon does not always correlate with the boundary of the domain, and the shuffling of these exons provides a stretch of amino acid sequences between the domains (Supplemental Table 5), these data suggest that most of the new domain pairs were acquired by exon shuffling. Supporting this idea, for more than half of the domains, the introns forming their boundaries have symmetrical phase combinations, and they can be inserted without disrupting the reading frame of the ancestral genes (Table 3 ). In addition, many of the domains are abutted by phase 1 introns at both of their boundaries, while phase 0 introns are dominant in the entire genomes of human and amphioxus (Fig. 3; Table 3; Supplemental Table 5). This is consistent with a previous study in which large numbers of introns participating in exon shuffling are phase 1 (Patthy 1999). Note also that while the novel domain is inserted at either the $\mathrm{N}$ or $\mathrm{C}$ terminus in most cases (Ekman et al. 2007), we found one clear example in which

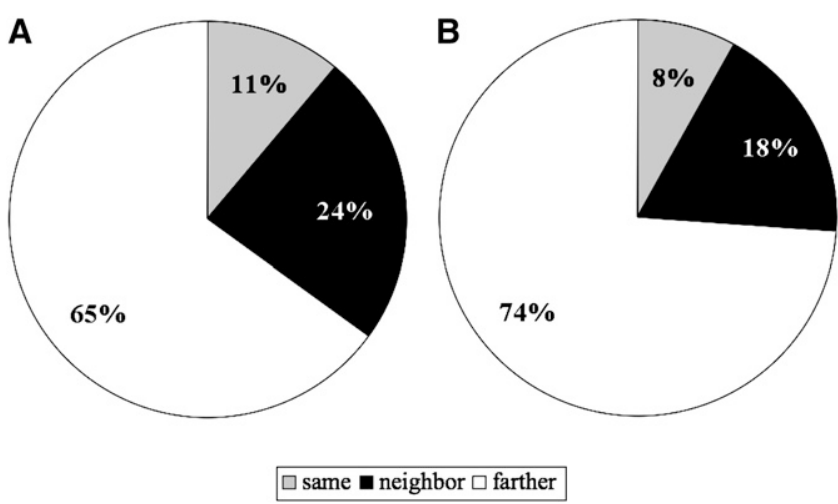

Figure 2. Position of the domain sequences relative to introns. (Neighbor) Domains are encoded by exons separated by one intron, (farther) domains are encoded by exons separated by one or more exons and two or more introns, (same) domains are encoded by the same exon. $(A)$ Seventy-five class II pairs of the vertebrates (Table 2 ) were found in 143 gene models in the human genome. The relative position of the domain sequences in these gene models is summarized. Details of the data are given in Supplemental Table 5. (B) Thirty-one class II pairs of the chordates (Table 4) were found in 76 gene models in the human genome. The relative position of the domain sequences in these gene models is summarized. Details of the data are given in Supplemental Table 7.
Table 3. Intron phases of the domains

\begin{tabular}{|c|c|c|c|c|}
\hline \multirow{2}{*}{$\begin{array}{l}\text { Intron phase } \\
\text { pair-type }\end{array}$} & \multicolumn{2}{|c|}{$\begin{array}{c}\text { Vertebrate-specific } \\
\text { Class II }\end{array}$} & \multicolumn{2}{|c|}{$\begin{array}{c}\text { Chordate-specific } \\
\text { Class II }\end{array}$} \\
\hline & Observed & $P$-value & Observed & $P$-value \\
\hline $0-0$ & 7 & 1.00 & 15 & 0.974 \\
\hline $1-1$ & 115 & 0 & 41 & 0 \\
\hline $2-2$ & 2 & 0.997 & 4 & 0.735 \\
\hline $0-1$ & $3 \overline{1}$ & 0.566 & 15 & 0.637 \\
\hline $0-2$ & 6 & 1.00 & 4 & 0.989 \\
\hline $1-2$ & 9 & 0.954 & 1 & 0.995 \\
\hline $1-0$ & 29 & 0.709 & 11 & 0.923 \\
\hline $2-0$ & 5 & 1.00 & 10 & 0.653 \\
\hline $2-1$ & 13 & 0.735 & 10 & 0.214 \\
\hline Total & 217 & & 111 & \\
\hline
\end{tabular}

Statistical analyses were performed based on the observed intron phases in human genomes (Phase 0, 66,882; Phase 1, 46,696; Phase 2, 32,260; total: 145,838$)$.

a new domain was inserted between the domains of the ancestral gene (Supplemental Fig. 2).

Among these class II domain pairs, some were obviously involved in the evolution of vertebrate-specific characteristics. As an example, aggrecan-the most abundant noncollagenous protein in cartilage-was created by domain shuffling in ancestral vertebrates, as has been previously suggested (Upholt et al. 1994; Valhmu et al. 1995; Patthy 2003). Aggrecan consists of five types of domains, and the combination of the immunoglobulin domain (V-set: PF07686; ig: PF00047) and extracellular link domain (Xlink: PF00193) was identified as a novel domain pair (Fig. 1E). Aggrecan is a heavily glycosylated protein. Repeated amino acid sequences that are modified with keratan sulfate and chondroitin sulfate moieties are located between the Xlink domain and the EGF domain (Fig. 1E), although these repeats were not recognized as protein motifs in the Pfam database. The Xlink domains are essential for binding with glycosaminoglycan hyaluronic acid. The molecular complex of hyaluronic acid and heavily glycosylated aggrecan provides the tensile strength that allows cartilage to absorb shock and resist compression in joints, and mutations in aggrecan cause severe cartilage defects and result in dwarfism (Watanabe and Yamada 1999).

To trace the evolutionary history of aggrecan genes, we examined each domain in the amphioxus and ascidian genomes. Since the V-set, ig, Lectin C, EGF, and Sushi domains are relatively abundant in both genomes, we traced the origin of the Xlink domain. Two ascidian gene models and 20 amphioxus gene models contained Xlink domains. One of the ascidian gene models (Ci-link1) encoded a single Xlink domain and an F5/8 type $\mathrm{C}$ domain (PF00754), and is expressed in some blood cells in juveniles (Fig. 1F,G). In vertebrates, the Xlink domain is found not only in aggrecan and its paralogs, but also in CD44, TNFAIP6 (also known as TSG6), LYVE1, and stabilin 1. These proteins contain single Xlink domains (Fig. 1E) and are involved in lymphocyte migration (Ponta et al. 2003; Kzhyshkowska et al. 2006). Therefore, the molecular structure and function of Ci-link1 more closely resemble those of these single-link proteins than those of aggrecan. These results suggest that the Xlink domain is a component of surface molecules on blood cells in protochordate ancestors. Moreover, the Xlink domain was combined with other domains, such as the V-set domain, in a vertebrate ancestor, and the protein was used as a component of cartilage-a unique vertebrate structure. Cartilage acidic protein 1 was also created by combining 


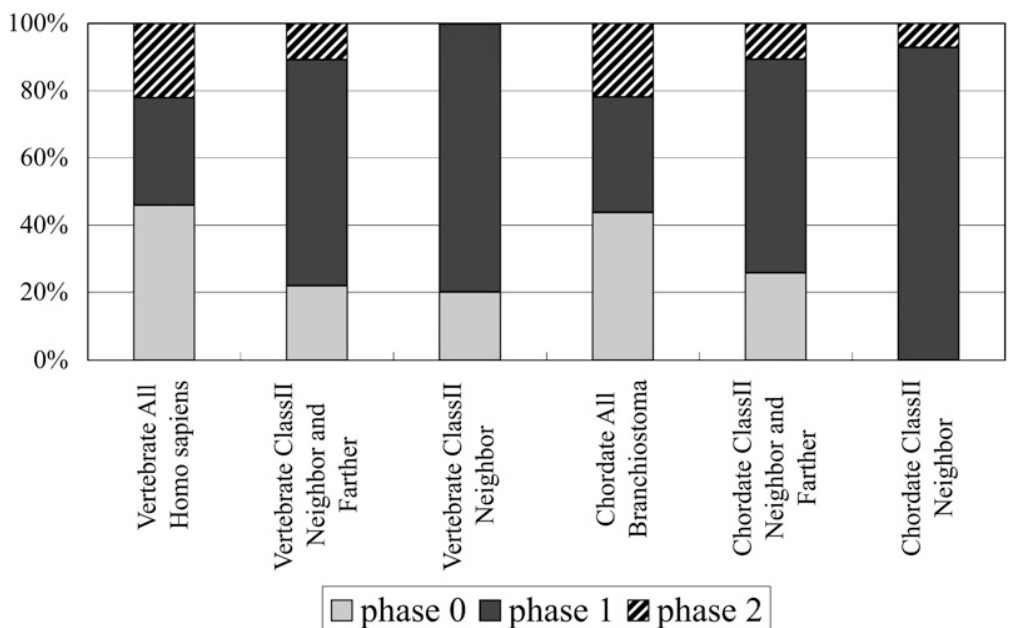

Figure 3. Phase of introns involved in domain shuffling. The intron phases of exons that encode the $C$ terminus of domain 1 and that of the $\mathrm{N}$ terminus of domain 2 were counted here. Although the phase 0 intron is dominant in the gene models of Homo and Branchiostoma, the phase 1 intron is dominant in the class II pairs. The intron phase of the class II pairs was analyzed using the human gene models. Details of the data are given in Supplemental Tables 5 and 7.

domains, including the ASPIC-and-UnbV (UnbV_ASPIC: PF07593) and calcium-binding EGF domains (EGF_CA: PF07645; Fig. 1H).

Among the vertebrate-specific domain pairs, we identified two proteins that contribute to cell junctions. Occludin-a component of tight junctions - contains a domain pair of a membraneassociating domain (MARVEL: PF01284) and an occludin_ELL domain (PF07303) that is specific to vertebrates (Supplemental Fig. 3). The amphioxus and ascidian proteomes possess an occludin ELL domain in the RNA polymerase II elongation factor ELL. Conversely, 17 amphioxus genes and four ascidian genes contain sequences encoding the MARVEL domain, and for each of these genes, the MARVEL domain was the only recognized functional domain. The structures of protochordate proteins containing the MARVEL domain were more similar to MAL and physins, which are involved in vesicle trafficking (Sánchez-Pulido et al. 2002). Therefore, a gene in an ancestral vertebrate acquired the sequence encoding the occludin_ELL domain, resulting in a new protein that functioned as a component of tight junctions, which are found only in vertebrates and ascidians together with the major tight junction component claudin. Interestingly, occludin is not an essential component of tight junctions, but rather has been suggested to be involved in the regulation of tight junctions by mediating extracellular cell adhesion signals (Saitou et al. 2000). Therefore, acquiring occludin as a component of tight junctions may have provided vertebrates with additional uses for these complexes. The gap junction protein, alpha 1, $43 \mathrm{kDa}$ (connexin43), was also found to contain a class I vertebrate-specific domain pair, which was composed of the Connexin (PF00029) and Connexin43 (PF03508) domains (Supplemental Fig. 3). The Connexin 43 domain, which is found only in vertebrates, serves as a regulatory domain for the gap junction protein alpha 1, $43 \mathrm{kDa}$ (connexin43) through its phosphorylation status (Axelsen et al. 2006).

Another notable class of genes that contained sequences encoding vertebrate-specific domain pairs included those involved in the vertebrate auditory system. Tectorin-alpha is a major component of the tectorial membrane in the mammalian inner ear; in humans, a missense mutation in the ZP domain of tectorin alpha causes hearing loss in the range of $50-80 \mathrm{~dB}$ (Verhoeve et al. 1998). Furthermore, a mutation in the LCCL domain of cochlin, a novel gene created by domain shuffling in an ancestral vertebrate, causes deafness (Supplemental Fig. 3; Robertson et al. 2006).

Note also that genes involved in neurogenesis and immune responses were also identified. Amyloid beta (A4) precursor and agrin are involved in synaptic physiology and synapse formation (Banks et al. 2003; Kamenetz et al. 2003), stabilin 1 is a scavenger receptor (Kzhyshkowska et al. 2006), and attractin is essential for initial immune cell clustering (Duke-Cohan et al. 1998). Complements $\mathrm{C} 6 / 7$ and $\mathrm{C} 3 / 4 / 5$ were also identified due to their unique combinations of domains (Azumi et al. 2003). Fibronectin, nidogen, and vitronectin were recognized as vertebrate-specific extracellular matrix (ECM) proteins among the class II domain pairs (Patthy 2003).

Although they may not have been achieved by domain shuffling, it is worth noting that various transcription factors (HOXC9, HNF1A, CDX2, NFIC, and ZFY) categorized as class I have acquired vertebrate-specific transactivation domains. It has been suggested that the evolution of the transactivation domain was critical for the evolution of arthropod leg morphogenesis (Galant and Carroll 2002).

\section{Domain shuffling in the common ancestors of chordates}

Of the 256 pairs acquired by the ancestral chordates (the last common ancestors of amphioxus, ascidians, and vertebrates), 43 pairs were conserved between the ascidian, amphioxus, and at least some of the vertebrate species. After surveying each domain pair in the CDD (http://www.ncbi.nlm.nih.gov/Structure/cdd/ cdd.shtml), 12 were found in other invertebrates (Supplemental Table 6) and were omitted from further analysis. The remaining 31 pairs (Table 4) were found in proteins encoded by 14 types of vertebrate genes. Sequences coding for all of these domains were found in nonchordate genomes; therefore, there were no class I pairs. Thirty-one pairs were found in 76 human gene models. Among these gene models, $18 \%$ were encoded by exons separated by one intron (referred to as "neighbor" in Fig. 2 and Supplemental Table 7) and $74 \%$ by exons separated by two or more introns and one or more exons ("farther" in Fig. 2 and Supplemental Table 7). Only $8 \%$ were encoded by the same exon ("same" in Fig. 2 and Supplemental Table 7). Many of the domains involved in chordate-specific pairs were also separated by phase 1 symmetric introns, and so these domain pairs were likely achieved by exon shuffling (Fig. 3; Table 3). In most cases, we confirmed that the protochordate genes listed in Table 4 encode the cognates of human genes by checking the best-hit genes in a BLASTP search.

Two unique domain pairs (the PF03098 An_peroxidase domain with Sushi or EGF) were encoded in the thyroid peroxidase gene, which is expressed in the endostyle or thyroid of chordates where it performs an essential role in iodide metabolism 


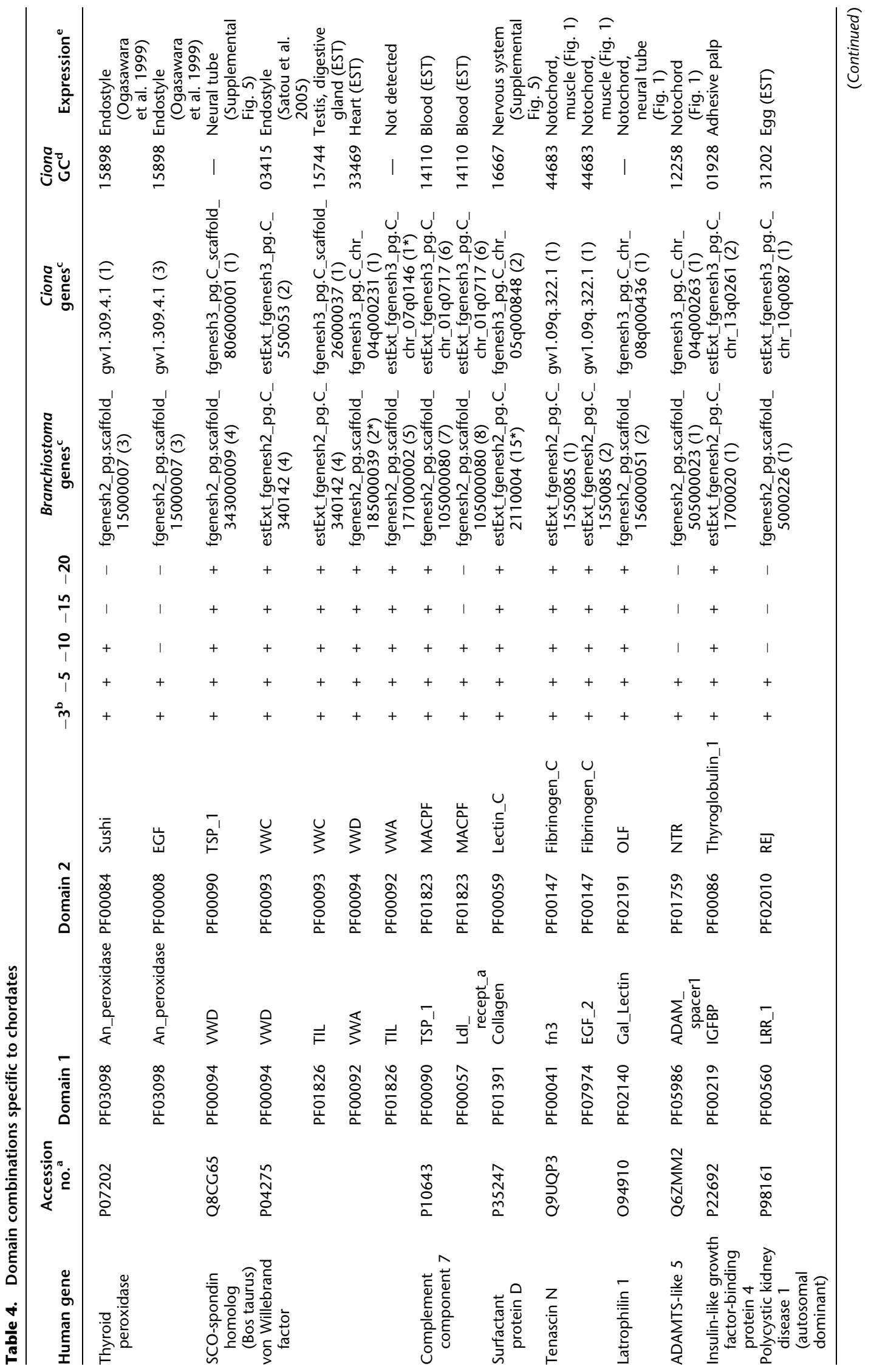




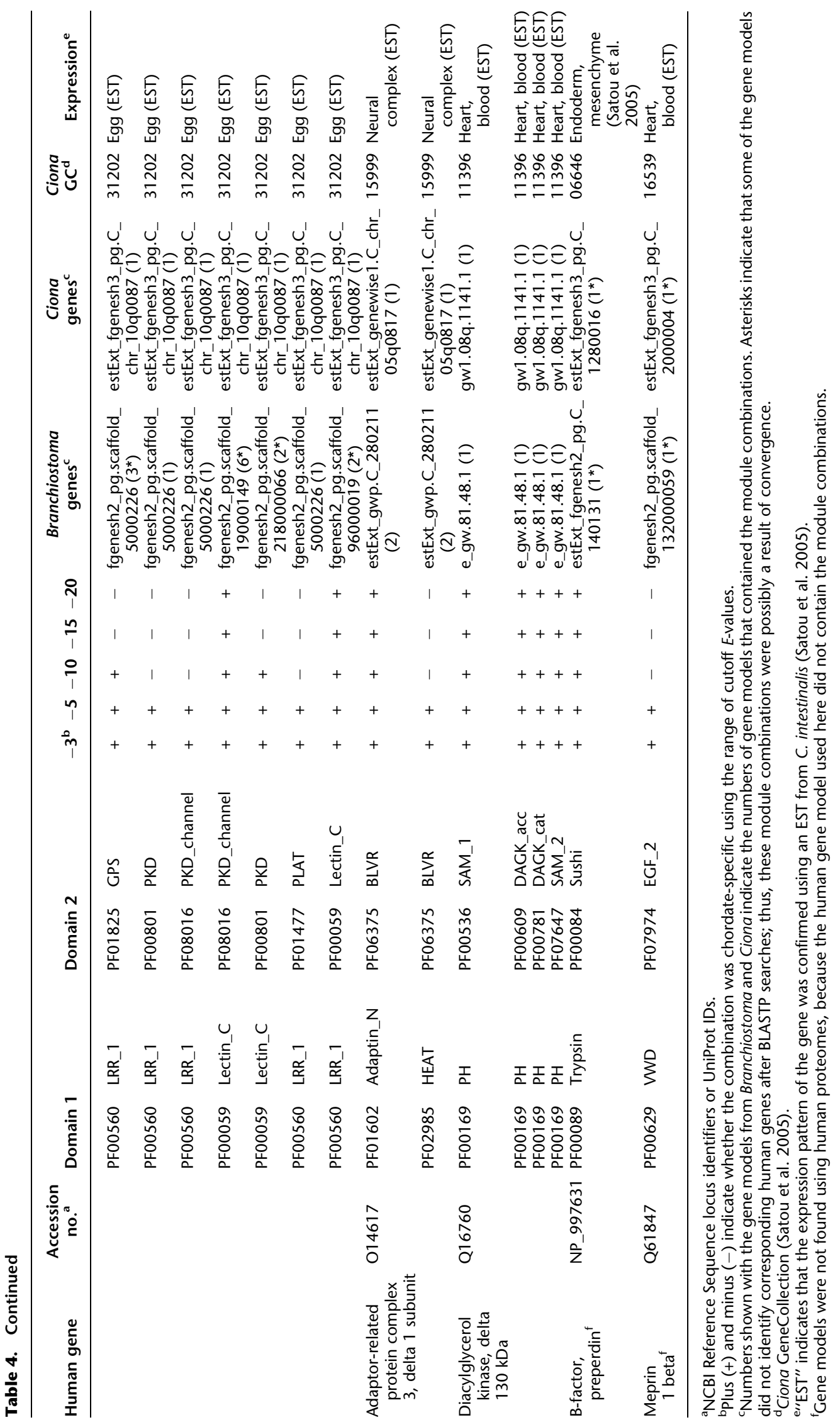


(Ogasawara et al. 1999). Since the endostyle/thyroid is a chordatespecific structure, it is likely that a gene with an essential role in the endostyle was created in ancestral chordates. The gene encoding the mucous protein VWF is also specific to chordates. Ciona VWF (Ciona gene collection ID 03415) has been reported to be expressed in the endostyle (Satou et al. 2005). vWF may be a component of mucus excreted from the endostyle, and originally functioned in the filter-feeding systems of chordates, which were subsequently used to form the blood-clotting machinery of vertebrates. The main component of Reissner's fiber, a chordatespecific structure that extends from the infundibular organ of the neural tube, is SCO-spondin (Gonçalves-Mendes et al. 2003), which was created by domain shuffling in chordates. Our results demonstrated that Ciona SCO-spondin was expressed in the neural tube (Supplemental Fig. 4).

The development of an innate immune system in chordates was facilitated by the expansion of protein repertoires. Our analysis recognized three domain pairs in two types of genes involved in innate immune systems as chordate-specific: complement component C6/7/8/9 and surfactant protein D (SP-D). Ciona C6/7/8/9 is expressed in the blood cells of ascidians and is also likely involved in their innate immune system (Satou et al. 2002, 2003). In contrast, the expression of Ciona $S P-D$ is detected only in the embryonic nervous system (Supplemental Fig. 4).

These examples indicate that, as in ancestral vertebrates, domain shuffling played important roles in the phenotypic evolution of ancestral chordates. In this study, we have taken advantage of the availability of the Ciona gene collection (http:// ghost.zool.kyoto-u.ac.jp/indexr1.html; Satou et al. 2005) to examine the expression of Ciona genes using in situ hybridizations. One of the important structures acquired by ancestral chordates was the notochord, which had an essential role in the formation of the chordate body plan (Satoh 2003). Therefore, it is notable that three of the identified ascidian genes, tenascin, latrophilin, and the ADAMTS-like 5 homolog, are expressed in the notochord (Fig. $1 \mathrm{~B}-\mathrm{D})$. Ciona tenascin is expressed in both the notochord and muscle (Fig. 1B), whereas vertebrate tenascin genes are expressed in a wide variety of cells, including the notochord and somites (Tongiorgi 1999). One of the original functions of tenascin may lie in the formation of the notochord. Latrophilin is thought to be involved in exocytosis (Lang et al. 1998), whereas ADAMTS-like protein 5 is a metalloprotease involved in ECM digestion (Tortorella et al. 2002). These proteins may have been essential for the evolution of notochord cells, which secrete the ECM that makes up the notochord sheath.

We made a list of domain pairs that were inferred to have been acquired by the common ancestors of vertebrates, as well as those acquired by common ancestors of chordates. These genes are likely to have gained new functional roles by acquiring new domains, and are likely to be involved in phenotypic evolution. Indeed, we have presented some examples showing that novel genes drove the evolution of the characteristic features of vertebrates or chordates.

Recently, comparative developmental biology revealed that the morphological features of various multicellular animals are built on a common set of genes, and morphological variation is attributable to the altered expression patterns of preexisting genes (Carroll et al. 2001). However, these studies are primarily concerned with transcription factors and signaling molecules, with less attention to structural proteins. We propose that, in addition to the regulatory change of preexisting genes, dramatic molecular evolutionary events such as the evolution of novel genes via domain shuffling have contributed to phenotypic evolution.

\section{Methods}

We used the Pfam database (Finn et al. 2006; Pfam_ls, release 20.0; http://pfam.sanger.ac.uk), which contains 8296 conserved domains. Protein entries matching conserved domains in Pfam were identified using HMMER searches (Eddy 1998) of a set of 20 species with complete genomic sequences. Details of the data sources are presented in Supplemental Table 1. In total, 4649 of 7677 Pfam domains were found in these proteomes ( $E$-value cutoff of $10^{-3}$ ). Some scripts for parsing the data and for making pairwise domain matrixes were written using Ruby (http://www.ruby-lang.org) and BioRuby (http://bioruby.org/).

Our analyses did not consider the number of domains in each protein; regardless of whether domain A was present with a single copy of domain B or multiple copies of domain B, the pair of domain A and domain B was simply scored as present. We did, however, consider the order of the domains. A domain pair of A and $\mathrm{B}$ with A closer to the $\mathrm{N}$ terminus was treated as distinct from a pair with $\mathrm{B}$ closer to the $\mathrm{N}$ terminus. We examined domain pairs specific to vertebrates or chordates using HMMER searches and $E$-value cutoffs from $10^{-3}$ to $10^{-20}$, and decided to use $E$-value cutoffs of as high as $10^{-3}$ because some vertebrate-specific pairs were eliminated when the cutoff values were lower, as the amino acid sequences of some of the domains were less conserved in some species. For these cases, we still regarded the domain pairs as significant. In contrast, some domain pairs were eliminated when the cutoff values were higher, because some pairs became positive in an outgroup taxa. We did not regard these pairs as specific to vertebrates or chordates. Therefore, the specific domain pairs were identified using a cutoff value of $10^{-3}$, and sequence conservation was assessed by checking whether the pairs were still specific at lower cutoff values.

The convergent index (CI) was calculated by comparing the domain architecture between two gene models. When we defined $X$ as the number of specific domain(s) of gene model A, $Y$ as that of gene model $\mathrm{B}$, and $Z$ as the number of common domains between the two gene models, then $\mathrm{CI}=X \times Y /(Z+X \times Y)$. We compared the $\mathrm{CI}$ between all gene models that had the domain pairs. When the lowest CI was $\geq 0.5$, we regarded the pairs as likely to have been acquired convergently (Supplemental Fig. 1).

We examined the expression of Ciona genes in the tailbud and young juvenile stages of all of the gene models listed in Table 4 except those whose expression had already been published. For those in which early expression was not detected, we list information on their expression from previously published studies. In situ hybridization was performed as described (Ogasawara et al. 2002; Satou et al. 2005).

\section{Acknowledgments}

We thank Seb Shimeld and Peter Holland for their critical reading of the manuscript and helpful comments. This work was supported by KAKENHI (Grant-in-Aid for Scientific Research) on Priority Areas "Comparative Genomics" from the Ministry of Education, Culture, Sports, Science and Technology of Japan to H.W. and N.S. T.K. was supported by JSPS Postdoctral Fellowships for Research Abroad. D.S.R. and N.H.P. were supported by CIG. D.S.R. was supported by R.A. Melon.

\section{References}

Axelsen LN, Stahlhut M, Mohammed S, Larsen BD, Nielsen MS, HolsteinRathlou N-H, Andersen S, Jensen ON, Hennan JK, Kjølbye AL. 2006. 
Identification of ischemia-regulated phosphorylation sites in connexin43: A possible target for the antiarrhythmic peptide analogue rotigaptide (ZP123). J Mol Cell Cardiol 40: 790-798.

Azumi K, De Santis R, De Tomaso A, Rigoutsos I, Yoshizaki F, Pinto M, Marino R, Shida K, Ikeda M, Ikeda M, et al. 2003. Genomic analysis of immunity in a Urochordate and the emergence of the vertebrate immune system: "Waiting for Godot." Immunogenetics 55: 570-581.

Babushok DV, Ostertag EM, Kazazian HH Jr. 2007. Current topics in genome evolution: Molecular mechanisms of new gene formation. Cell Mol Life Sci 64: 542-554.

Banks G, Choy P, Lavidis N, Noakes P. 2003. Neuromuscular synapses mediate motor axon branching and motoneuron survival during the embryonic period of programmed cell death. Dev Biol 257: 71-84.

Carroll SB, Greiner JK, Weatherbee SD. 2001. From DNA to diversity. Blackwell Science, Malden, MA.

Chothia C, Gough J, Vogel C, Teichmann S. 2003. Evolution of the protein repertoire. Science 300: 1701-1703.

Davidson EH. 2006. The regulatory genome. Academic Press, San Diego, CA.

Dehal P, Satou Y, Campbell PK, Chapman J, Degnan B, De Tomaso A, Davidson B, Di Gregorio A, Gelpke M, Goodstein DM, et al. 2002. The draft genome of Ciona intestinalis: Insights into chordate and vertebrate origins. Science 298: 2157-2167.

Duke-Cohan JS, Gu J, McLaughlin DF, Xu Y, Freeman GJ, Schlossman SF. 1998. Attractin (DPPT-L), a member of the CUB family of cell adhesion and guidance proteins, is secreted by activated human T lymphocytes and modulates immune cell interactions. Proc Natl Acad Sci 95: 1133611341.

Eddy SR. 1998. Profile hidden Markov models. Bioinformatics 14: 755-763.

Ekman D, Bjorkund A, Elofsson A. 2007. Quantification of the elevated rate of domain rearrangements in Metazoa. J Mol Biol 372: 1337-1348.

Finn RD, Mistry J, Schuster-Böckler B, Griffiths-Jones S, Hollich V, Lassmann T, Moxon S, Marshall M, Khanna A, Durbin R, et al. 2006. Pfam: Clans, web tools and services. Nucleic Acids Res 34: D247-D251.

Galant R, Carroll SB. 2002. Evolution of a transcriptional repression domain in an insect Hox protein. Nature 415: 910-913.

Goncalves-Mendes N, Simon-Chazottes D, Creveaux I, Meiniel A, Guénet J-L, Meiniel R. 2003. Mouse SCO-spondin, a gene of the thrombospondin type 1 repeat (TSR) superfamily expressed in the brain. Gene 312: 263-270.

Gough J. 2005. Convergent evolution of domain architectures (is rare). Bioinformatics 21: 1464-1471.

Holland PW, Garcia-Fernandez J, Williams NA, Sidow A. 1994. Gene duplications and origins of vertebrate development. Development 1994 (Suppl.): 125-133.

Holland, LZ, Abalat R, Azumi K, Benito-Gutiérrez E, Blow MJ, BronnerFraser M, Brunet F, Butts T, Candiani S, Dishaw LJ, et al. 2008. The amphioxus genome illuminates vertebrate origins and cephalochordate biology. Genome Res 18: 1100-1111.

Huang S, Yuan S, Guo L, Yu Y, Li J, Wu T, Liu T, Yang M, Wu K, Liu H, et al. 2008. Genomic analysis of the immune gene repertoire of amphioxus reveals extraordinary innate complexity and diversity. Genome Res 18: 1112-1126.

Kaessmann H, Zollner S, Nekrutenko A, Li W. 2002. Signatures of domain shuffling in the human genome. Genome Res 12: 1642-1650.

Kamenetz F, Tomita T, Hsieh H, Seabrook G, Borchelt D, Iwatsubo T, Sisodia S, Malinow R. 2003. APP processing and synaptic function. Neuron 37: 925-937.

Kzhyshkowska J, Gratchev A, Goerdt S. 2006. Stabilin-1, a homeostatic scavenger receptor with multiple functions. J Cell Mol Med 10: 635-649.

Lang J, Ushkaryov Y, Grasso A, Wollheim CB. 1998. Ca ${ }^{2+}$-independent insulin exocytosis induced by $\alpha$-latrotoxin requires latrophilin, a $\mathrm{G}$ protein-coupled receptor. EMBO J 17: 648-657.

Lee YH, Huang GM, Cameron RA, Graham G, Davidson EH, Hood L, Britten RJ. 1999. EST analysis of gene expression in early cleavage-stage sea urchin embryos. Development 126: 3857-3867.
Liu M, Grigoriev A. 2004. Protein domains correlate strongly with exons in multiple eukaryotic genomes: Evidence of exon shuffling? Trends Genet 20: $399-403$.

Ogasawara M, Lauro RD, Satoh N. 1999. Ascidian homologs of mammalian thyroid peroxidase genes are expressed in the thyroid-equivalent region of the endostyle. J Exp Zool 285: 158-169.

Ogasawara M, Sasaki A, Metoki H, Shin-i T, Kohara Y, Satoh N, Satou Y. 2002. Gene expression profiles in young adult Ciona intestinalis. Dev Genes Evol 212: 173-185.

Patthy L. 1999. Genome evolution and the evolution of exon-shuffling-A review. Gene 238: 103-114.

Patthy L. 2003. Modular assembly of genes and the evolution of new functions. Genetica 118: 217-231.

Ponta H, Sherman L, Herrlich PA. 2003. CD44: From adhesion molecules to signalling regulators. Nat Rev Mol Cell Biol 4: 33-45.

Putnam NH, Butts T, Ferrier DEK, Furlong, RF, Hellsten, U, Kawashima T, Robinson-Rechavi M, Shoguchi E, Terry A, Yu J-K, et al. 2008. The amphioxus genome and the evolution of the chordate karyotype. Nature 453: 1064-1071.

Robertson N, Hamaker S, Patriub V, Aster J, Morton C. 2006. Subcellular localisation, secretion, and post-translational processing of normal cochlin, and of mutants causing the sensorineural deafness and vestibular disorder, DFNA9. J Med Genet 40: 479-486.

Saitou M, Furuse M, Sasaki H, Schulzke J-D, Fromm M, Takano H, Noda T, Tsukita S. 2000. Complex phenotype of mice lacking occludin, a component of tight junction strands. Mol Biol Cell 11: 4131-4142.

Sánchez-Pulido L, Martín-Belmonte F, Valencia A, Alonso MA. 2002. MARVEL: A conserved domain involved in membrane apposition events. Trends Biochem Sci 27: 599-601.

Satoh N. 2003. The ascidian tadpole larva: Comparative molecular development and genomics. Nat Rev Genet 4: 285-295.

Satou Y, Yamada L, Mochizuki Y, Takatori N, Kawashima T, Sasaki A, Hamaguchi M, Awazu S, Yagi K, Sasakura Y, et al. 2002. A cDNA resource from the basal chordate Ciona intestinalis. Genesis 33: 153-154.

Satou Y, Kawashima T, Kohara Y, Satoh N. 2003. Large scale EST analyses in Ciona intestinalis, its application as Northern blot analyses. Dev Genes Evol 213: 314-318.

Satou Y, Kawashima T, Shoguchi E, Nakayama A, Satoh N. 2005. An integrated database of the ascidian, Ciona intestinalis: Towards functional genomics. Zoolog Sci 22: 837-843.

Sea Urchin Genome Sequencing Consortium. 2006. The genome of the sea urchin Strongylocentrotus purpuratus. Science 314: 941-952.

Tongiorgi E. 1999. Tenascin-C expression in the trunk of wild-type, cyclops and floating head zebrafish embryos. Brain Res Bull 48: 79-88.

Tortorella MD, Liu R-Q, Burn T, Newton RC, Arner E. 2002. Characterization of human aggrecanase 2 (ADAM-TS5): Substrate specificity studies and comparison with aggrecanase 1 (ADAM-TS4). Matrix Biol 21: 499511.

Upholt WB, Chandrasekaran L, Tanzer ML. 1994. Molecular cloning and analysis of the protein modules of aggrecans. EXS 70: $37-52$.

Valhmu WB, Palmer GD, Rivers PA, Ebara S, Cheng JF, Fischer S, Ratcliffe A. 1995. Structure of the human aggrecan gene: Exon-intron organization and association with the protein domains. Biochem J 309: 535-542.

Verhoeve K, Van Laer L, Kirschhofer K, Legan P, Hughes D, Schatteman I, Verstreken M, Van Hauwe P, Coucke P, Chen A, et al. 1998. Mutations in the human $\alpha$-tectorin gene cause autosomal dominant non-syndromic hearing impairment. Nat Genet 19: 60-62.

Vibranovski MD, NJ Sakabe, de Oliveira RS, de Souza SJ. 2005. Signs of ancient and modern exon-shuffling are correlated to the distributuon of ancient and modern domains along proteins. J Mol Evol 61: 341-350.

Watanabe H, Yamada Y. 1999. Mice lacking link protein develop dwarfism and craniofacial abnormalities. Nat Genet 21: 225-229.

Received September 23, 2008; accepted in revised form April 24, 2009. 


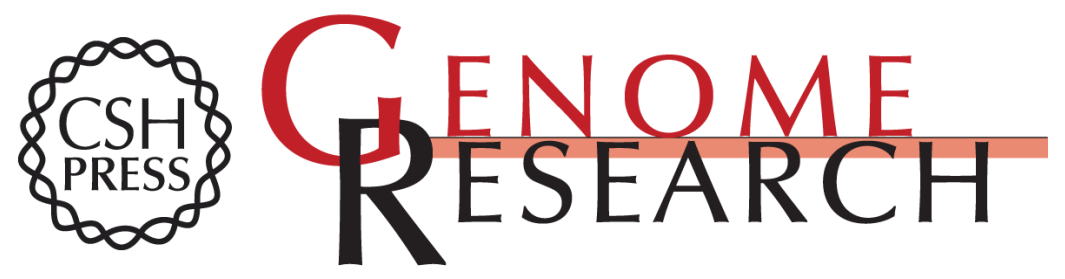

\section{Domain shuffling and the evolution of vertebrates}

Takeshi Kawashima, Shuichi Kawashima, Chisaki Tanaka, et al.

Genome Res. 2009 19: 1393-1403 originally published online May 14, 2009

Access the most recent version at doi:10.1101/gr.087072.108

\section{Supplemental http://genome.cshlp.org/content/suppl/2009/06/17/gr.087072.108.DC1 \\ Material}

References This article cites 43 articles, 12 of which can be accessed free at:

http://genome.cshlp.org/content/19/8/1393.full.html\#ref-list-1

\section{License}

Email Alerting Receive free email alerts when new articles cite this article - sign up in the box at the Service top right corner of the article or click here.

\section{Affordable, Accurate Sequencing.}

